\title{
Análisis de métodos OMA para la extracción de parámetros modales sobre edificios existentes
}

\section{Analysis of OMA methods for the extraction of modal parameters on existing buildings}

\author{
Víctor Samaniego Galindo ${ }^{1}$ https://orcid.org/0000-0002-6105-0592, Iván Palacios Serrano ${ }^{2}$ \\ https://orcid.org/0000-0002-3894-3341, José Placencia León² https://orcid.org/0000-0001-8036-6798, Milton \\ Muñoz Calle ${ }^{2}$ https://orcid.org/0000-0001-6663-3140, Santiago González Martínez ${ }^{2}$ \\ https://orcid.org/0000-0001-6604-889X , Juan Jiménez Pacheco 1 https://orcid.org/0000-0002-8977-9879
}

${ }^{1}$ Universidad de Cuenca, Departamento de Ingeniería Civil, Cuenca, Ecuador victor.samaniegog@ucuenca.edu.ec, juan.jimenez@ucuenca.edu.ec
${ }^{2}$ Universidad de Cuenca, Departamento de Ingeniería Eléctrica, Electrónica y Telecomunicaciones, Cuenca, Ecuador ivan.palacios@ucuenca.edu.ec, jose.placencial@ucuenca.edu.ec, milton.munozc@ucuenca.edu.ec, santiago.gonzalezm@ucuenca.edu.ec

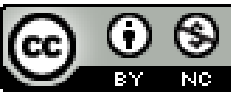

Esta obra está bajo una licencia internacional Creative Commons Atribución-NoComercial 4.0.

$\begin{array}{ll}\text { Enviado: } & 2021 / 06 / 26 \\ \text { Aceptado: } & 2021 / 09 / 28 \\ \text { Publicado: } & 2021 / 11 / 30\end{array}$

\section{Resumen}

En este artículo se presenta la aplicación de métodos de análisis modal operacional (OMA, Operational Modal Analysis) con el objetivo de caracterizar los parámetros modales (v.g. frecuencias y modos de vibración) de una edificación. El estudio se realiza sobre un escenario real consistente en un edificio esencial. En concreto se emplea: el método de descomposición en el dominio de la frecuencia (FDD, Frequency Domain Decomposition) y su versión mejorada (EFDD, Enhanced-FDD). En una primera etapa, se lleva a cabo una evaluación estructural preliminar del edificio (empleando el método de inspección visual rápida, RVS), un levantamiento de dimensiones y ensayos en campo para la caracterización mecánica de sus componentes, todo ello con el propósito de conseguir un análisis modal convencional lo más fiable posible en términos de sus parámetros modales. Con base en este análisis modal, se diseña un plan de instrumentación con acelerómetros triaxiales de sistemas microelectromecánicos (MEMS); el proceso de instrumentación abarca tres etapas: la adquisición, el control y el almacenamiento de información. La principal contribución de este trabajo consiste en la evaluación de la aplicación de los métodos FDD y EFDD sobre un edificio esencial, con la particularidad del uso de vibraciones de microsismicidad para la identificación de parámetros modales. El análisis de los resultados obtenidos determina una

Sumario: Introducción, Metodología, Resultados y Conclusiones.

Como citar: Samaniego, V., Palacios, I., Placencia, S., Muñoz, M., González, S. \& Jiménez, J. (2021). Análisis de métodos OMA para la extracción de parámetros modales sobre edificios existentes. Revista Tecnológica - Espol, 33(2), 75-93. http://www.rte.espol.edu.ec/index.php/tecnologica/article/view/832 
frecuencia fundamental del edificio de $1.43 \mathrm{~Hz}$, y evidencia un comportamiento modal no recomendado.

Palabras clave: acelerógrafos, EFDD, FDD, instrumentación, salud estructural.

\begin{abstract}
This paper presents the application of Operational Modal Analysis (OMA) methods in order to characterize the modal parameters (i.e., frequencies and vibration modes) of a building. The study was carried out on a real scenario. In particular, the Frequency Domain Decomposition (FDD) method and its enhanced version (EFDD) were used. In a first stage, a preliminary structural evaluation of the building (using the rapid visual inspection method, RVS), a dimensional survey and field tests for the mechanical characterization of its components were carried out, for the purpose of obtaining a conventional modal analysis in terms of its modal parameters. Based on this modal analysis, an instrumentation plan was designed with triaxial MEMS accelerometers (microelectromechanical systems). The instrumentation process was comprised of three stages: acquisition, control and storage of information. The main contribution of this work consists of the evaluation of the application of the FDD and EFDD methods on an essential building, with the particularity of the use of microseismicity vibrations for the identification of modal parameters. Results obtained reveal a fundamental frequency of the building of $1.43 \mathrm{~Hz}$, as well as a non-recommended modal behavior.
\end{abstract}

Keywords: accelerographs, EFDD, FDD, instrumentation, structural health.

\title{
Introducción
}

Esta investigación presenta la caracterización de los parámetros modales de una edificación (frecuencias y modos de vibración) por medio de la monitorización de salud estructural (SHM, Structural Health Monitoring), orientado a la detección y alerta temprana de daños en una estructura. La monitorización de salud estructural es una estrategia de evaluación estructural basada en el análisis de vibraciones y orientada hacia la detección y localización de daños en las estructuras (Kavitha et al., 2016). En tal contexto, la detección temprana de daño estructural ayuda al mantenimiento de la estructura y prevención de eventos catastróficos; esto supone un ahorro económico a largo plazo. Con respecto a las aplicaciones de la monitorización de salud estructural, en (Boscato et al., 2016) y (Muttillo et al., 2019) se propone el análisis de edificaciones a través del despliegue de sensores estratégicamente ubicados. Por otro lado, en (Pachón et al., 2018) se realiza el estudio de la salud estructural del puente de Posadas (Córdoba, España) con la metodología OSP (Optimal Sensor Placement) para una eficiente monitorización continua del puente, obteniéndose errores menores al $2 \%$. En otro ámbito de aplicaciones, en (Neu et al., 2016) se analiza el estado de las alas de un avión debido a los flujos transónicos a los que están expuestas.

Como etapa preliminar del proceso de recopilación de información, es necesario realizar un levantamiento estructural. Para esto, de gran utilidad resultan los lineamientos definidos en el documento FEMA 154 (Rojahn et al., 2002), centrado en el método RVS (Rapid Visual Screening). Este procedimiento comprende las tareas de revisión de planos existentes, recolección de información dimensional de elementos estructurales, detección de fallas y patologías en el edificio.

A continuación, se desarrolla la caracterización mecánica de los elementos de hormigón de la estructura. Finalmente, a partir de un modelo elástico-lineal se desarrolla un análisis modal convencional (mediante el uso de programas de análisis estructural como 
ETABS); el comportamiento modal obtenido guía el plan de instrumentación sobre el edificio. Este análisis se realiza previamente en (Samaniego, 2020).

En el marco de los métodos de análisis modal operacional (OMA, Operational Modal Analysis), la instrumentación se implementa, en general, con acelerómetros. Por ejemplo, en (Valenti et al., 2018) se propone un sistema SHM basado en acelerómetros de tipo MEMS (Microelectromechanical Systems); dicho sistema basa su funcionamiento en las etapas de adquisición, control, almacenamiento y transmisión de datos. Además, la ubicación adecuada de los sensores en la estructura es fundamental para la correcta instrumentación sísmica. En este contexto, en (Ostachowicz et al., 2019) se analizan diferentes métodos de colocación óptima de sensores (OSP, Optimal Sensor Placement) así como el desarrollo de sus algoritmos. De forma similar, en (Pachón et al., 2020) se estudian múltiples técnicas de colocación óptima de sensores en el Monasterio de San Jerónimo de Buenavista (Sevilla, España), donde se define el número de sensores y el método más eficiente para dicho caso de estudio.

A partir de los datos recolectados por los sensores, es posible aplicar uno o más de los métodos OMA con miras a la caracterización dinámica de una estructura mediante el análisis de sus modos de vibración (Greiner, 2009). Así pues, existen diferentes métodos de OMA, tanto en el dominio de la frecuencia como del tiempo, como, por ejemplo, el método Peak Picking (PP), el método ERA (Eigensystem Realization Algorithm) y la técnica de Identificación de Subespacios Estocásticos (SSI).

En la literatura, se describen diversos estudios que proponen la aplicación de métodos OMA para evaluar el estado de una estructura. Así, en (Cárdenas \& Medina, 2021) se describen los métodos de descomposición en el dominio de la frecuencia (FDD) y método mejorado de descomposición en el dominio de la frecuencia (EFDD) en un caso de estudio; los resultados presentaron una buena correlación en el caso de frecuencias y una correlación baja para las formas modales. Con el objetivo de obtener los modos de vibración y las frecuencias naturales de una estructura, en (Gkoktsi \& Giaralis, 2019) se aplica el método FDD y la técnica de muestreo ciego del espectro de potencia (PSBS, Power Spectrum Blind Sampling). En (Yun et al., 2021) se estudia el método EFDD para estructuras de gran altura en uso o en construcción, con los resultados de este análisis se propone un método alternativo de análisis denominado MCEFDD (Minimum Conditions for an Enhanced Frequency Domain Decomposition). Finalmente, en (Lacanna et al., 2016) se describe un procedimiento automático para obtener parámetros modales en tiempo real mediante el método EFDD; dicho estudio se realiza en el edificio del Baptisterio de San Giovanni, en Florencia.

En este país los estudios de aplicación de métodos de OMA en el ámbito de la ingeniería de estructuras aún son muy escasos (Arcentales, 2020). En tal contexto, con el objetivo de evaluar el comportamiento de una estructura ante eventos de cualquier tipo que pueden provocar daño, el presente trabajo desarrolla una aplicación del OMA sobre un edificio esencial de la ciudad de Cuenca, aplicado al edificio matriz de la Empresa Eléctrica Regional Centro Sur (Cuenca-Ecuador). La aplicación de OMA se organiza en las siguientes etapas: 1) levantamiento estructural, 2) caracterización de materiales, 3) ubicación de los sensores acelerómetros, 4) desarrollo e instalación de la instrumentación sísmica, 5) adquisición de datos de los sensores y 6) procesamiento de datos mediante los métodos FDD y EFDD (de OMA). Como resultado, se estiman ciertos parámetros modales y se determina el comportamiento del edificio ante la presencia de eventos. Esto permite obtener las zonas de la estructura propensas a sufrir daños en caso de que ocurra un evento sísmico. Este análisis facilita la intervención de manera temprana de dichas zonas y, en consecuencia, la prevención de daños irreversibles y conservación de la edificación. 
La principal contribución de esta investigación corresponde a la elaboración de una metodología orientada al análisis del comportamiento de una edificación frente a eventos que provocan daño en la estructura, por medio del OMA. Se elabora un plan de instrumentación y se definen zonas a instrumentarse de manera eficiente en términos de cantidad de sensores, datos representativos y bajos costos. Además, se define un proceso de análisis mediante dos métodos en el dominio de la frecuencia y se valora su importancia en función de la correlación de los parámetros modales obtenidos.

El presente artículo se divide en tres secciones. La primera, desarrolla la metodología empleada para la monitorización de salud estructural del edificio objeto de estudio. En ella se detalla el estudio estructural, la arquitectura tecnológica y el procesamiento de datos mediante los métodos FDD y EFDD para el análisis modal. En la segunda sección se describen y examinan los resultados obtenidos del análisis OMA, específicamente los parámetros modales. Finalmente, se presentan las conclusiones.

\section{Metodología}

Esta sección describe la metodología empleada. Específicamente, las etapas de análisis estructural, arquitectura tecnológica y procesamiento de datos. Con respecto a la primera etapa se describe el proceso de levantamiento estructural del edificio, el cual incluye la caracterización mecánica de sus elementos de hormigón (resistencias a compresión); además, a partir de los resultados del análisis modal numérico del edificio, esta primera etapa culmina con el establecimiento de su plan de instrumentación. En la segunda etapa se analiza la arquitectura tecnológica implementada en la investigación. Finalmente, en la tercera etapa se define la metodología usada en el análisis modal operacional (OMA) para el procesamiento de datos y estimación de los parámetros modales de la estructura.

\section{Análisis Estructural}

\section{Levantamiento Estructural}

En la Figura 1, se presenta el procedimiento desarrollado para el levantamiento estructural del edificio, el cual es una evaluación y recopilación in-situ de información del edificio. Como se observa, se desarrolla según los lineamientos planteados en el documento FEMA 154 (Rojahn et al., 2002). En este proceso se usa el método denominado Rapid Visual Screening (RVS), el cual se adapta al estudio en función de los parámetros que se deseen obtener. Dicho método está conformado por una secuencia que empieza por el desarrollo de un presupuesto, en función de la extensión de los exámenes a realizarse y el uso que se dará a los datos. Luego, se realiza una planificación del estudio y se define el área a examinarse. Una vez definida la planificación, se procede a seleccionar al equipo de trabajo, se agrupa y revisa la información recopilada previamente.

Posteriormente, con los planos de la edificación, se revisan medidas, la edad de la construcción y su tipología. Esta información se obtiene in-situ y se compara con los planos. Además, se realiza una inspección visual al interior y exterior de la construcción en planta y elevación, documentándola con fotografías, listas de chequeo y filmaciones; se examina la información recopilada y se elaboran registros.

De manera complementaria al método RVS, es necesario realizar mediciones de caracterización geométrica del edificio: longitudes de los vanos, alturas de entrepiso, áreas de bloques nuevos dimensiones de elementos (v.g. columnas, muros, losas, paredes de mampostería). En nuestro caso, estas mediciones se llevan a cabo utilizando un distanciómetro laser y cinta métrica; con esta información, se actualiza/valida (y en algunos casos, se corrige) la información contenida en los planos. 
Otro aspecto del proceso del levantamiento de información, con el fin de evaluar el estado estructural de un edificio, comprende la detección de distintos tipos de fallas en elementos estructurales y no estructurales de la edificación, tales como grietas, fisuras e hinchazones. La detección grietas y fisuras se realiza de manera visual mediante inspección de zonas vulnerables. La hinchazón de los elementos se visualiza con un nivel colocado transversalmente al elemento, de manera que se pueda evidenciar la presencia de una irregularidad en su superficie.

Además de la constatación/corrección de la información de planos y la evaluación del estado estructural, otra de las finalidades que persigue el levantamiento de información es la identificación tipológica del edificio, importante para su modelización numérica. Por último, con base en los datos recogidos, se proponen estudios o ensayos futuros necesarios para la recopilación de información adicional.

\section{Figura 1}

Proceso del Levantamiento Estructural del Edificio Objeto de Estudio

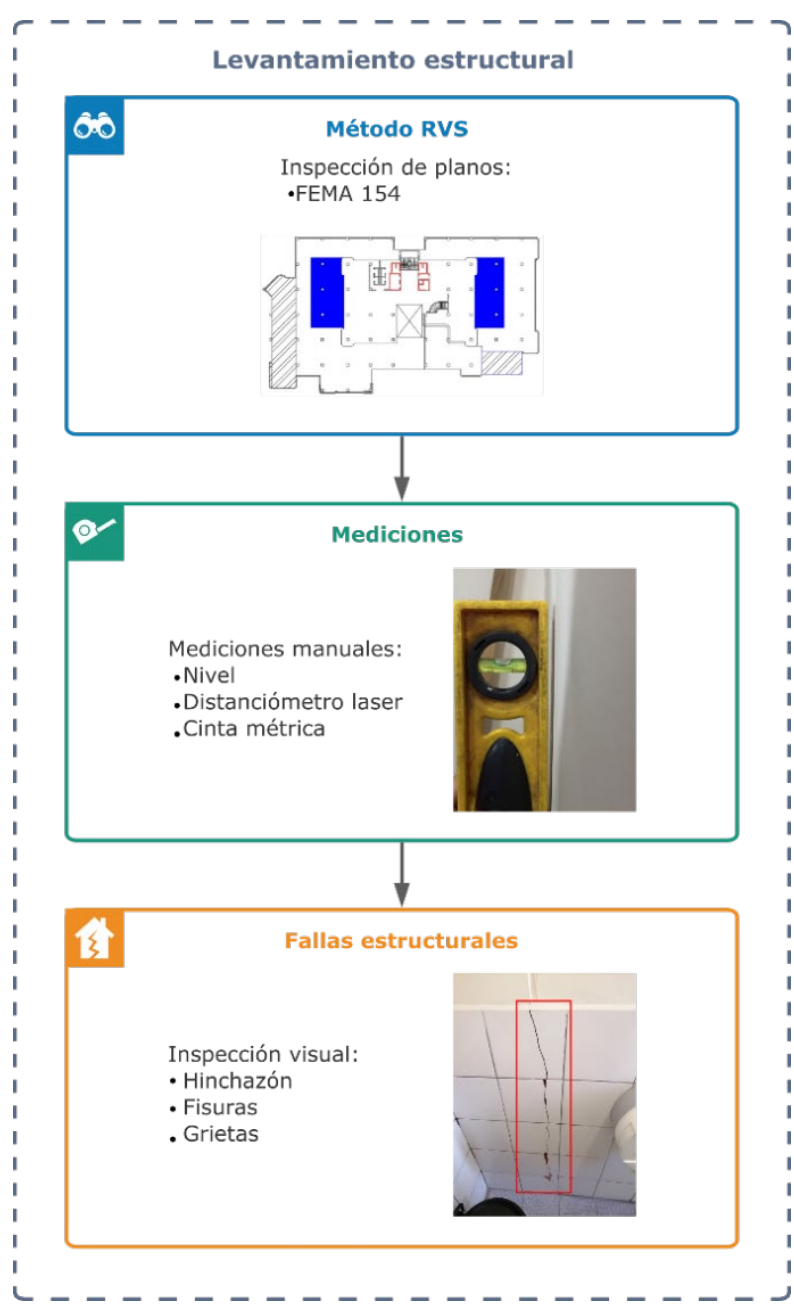

\section{Caracterización de Materiales}

En la Figura 2 se indica el proceso de caracterización del hormigón, material principal del sistema estructural del edificio, correspondiente a edificio de losa plana sobre columnas. La finalidad de este proceso es la de estimar el valor de la resistencia a compresión de los elementos de hormigón del edificio. A este propósito, se desarrollan 4 ensayos de tipo destructivo y no-destructivo. 
Para no comprometer el acero de refuerzo al llevar a cabo los ensayos de esclerometría y de extracción de núcleos de hormigón, se hace necesario un escaneo estructural (del hormigón) con el fin de detectar el refuerzo longitudinal y trasversal en los elementos de hormigón. Dicho estudio se realiza cuando se desconoce la ubicación y características de los aceros en los elementos estructurales de una edificación. Para la aplicación del ensayo se emplea el equipo Profometer 6 (PROCEQ SA, 2017).

En segunda instancia, se realizan ensayos de esclerometría (no-destructivos), los cuales estiman los valores de resistencia a compresión de un elemento in-situ, sin tener que extraer muestras o perforar el área de ensayo. Para ello, se utiliza el equipo martillo (PROCEQ SA, 2016) según el procedimiento descrito en la norma (ASTM C-805/C805M-18, 2018) y en (ACI 228.1R-03, 2003), la cual, cabe señalar, sugiere registrar al menos 10 lecturas válidas por elemento y descartar valores que difieran de la media en un valor mayor a 6 unidades. Estos datos son correlacionados con una curva definida por el técnico a base de la granulometría del hormigón ensayado; de esta forma, se relaciona el índice de rebote y la resistencia estimada.

\section{Figura 2}

\section{Proceso de Caracterización de Materiales de Hormigón y Ensayos In-situ}

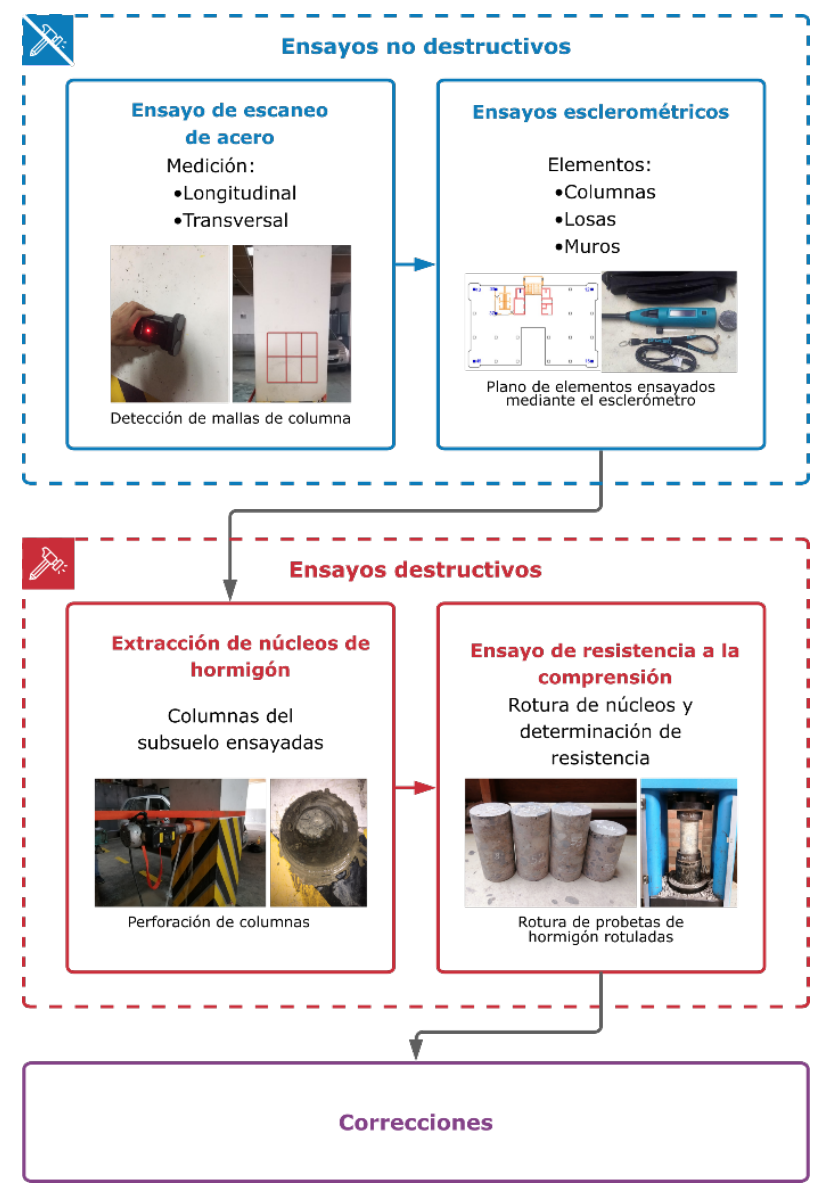

De manera complementaria, se realiza la extracción de núcleos de hormigón, este se utiliza para obtener probetas de hormigón in-situ, directamente del elemento en una edificación existente. Este ensayo de tipo destructivo consiste en extraer las muestras a base de los lineamientos descritos en la norma (ASTM C42 / C42M - 18, 2018) y posteriormente completar el ensayo con el relleno del orificio de extracción. 
Los cilindros de hormigón extraídos se ensayan a compresión en un laboratorio. Como se indica en la Figura 2, inicialmente se registran las medidas de altura, diámetro y peso de las muestras. Luego, estos son perfilados y cortados a las medidas normadas. Por último, se rompen los cilindros en la prensa hidráulica a base de la norma (ASTM C39 / C39M - 18, 2018) y de esta manera se obtiene el valor de resistencia a la compresión de los especímenes. Este ensayo de tipo destructivo permite obtener un factor de corrección para las resistencias estimadas en las pruebas esclerométricas. Para este objetivo se comparan y evalúan los resultados con los del ensayo esclerométrico. Posteriormente se obtienen las resistencias con el ensayo de resistencia a la compresión y se corrigen los resultados mediante una curva esclerométrica real de correlación.

\section{Plan de instrumentación}

El último proceso del análisis estructural consiste en diseñar e implementar un plan de instrumentación. El objetivo principal de un programa de instrumentación sísmica para sistemas estructurales es analizar el comportamiento y daño potencial de las estructuras bajo la acción de eventos que provoquen daño (Celebi et al., 1998).

Esta etapa comienza con un análisis modal numérico (convencional) de la estructura, mediante el cual se determinarán los parámetros modales del edificio. El modelo para el análisis modal se elabora en función de las características de la estructura obtenidos del levantamiento estructural. En este análisis se aplican los lineamientos planteados en (Chopra, 2013; Hsiao, 2009) así como en la Norma Ecuatoriana de Construcción en su apartado de peligro sísmico (NEC-SE-DS, 2015). Los resultados recogidos del análisis modal corresponden a los períodos de vibración, factores de participación modal y configuraciones de los modos de vibración para el edificio.

Con los periodos y modos de vibración, se ubican las zonas más vulnerables o con comportamientos deficientes en el edificio. Esta información sirve de insumo básico para el diseño del plan de instrumentación. En este sentido, autores como: (Boscato et al., 2016; Celebi, 2000; Celebi et al., 1998; Zhou et al., 2017) recomiendan tener sensores en la parte baja, centro y en la parte alta de la edificación. Además, la ubicación de los sensores en pisos adicionales depende de ciertas características del edificio como el número de pisos, la contribución de los modos de vibración y los cambios de rigideces entre pisos.

En función de las consideraciones mencionadas, se elabora un plan de instrumentación para el edificio con 6 alternativas, las cuales se basan en sensores axiales, biaxiales o triaxiales. Cada propuesta tiene como objetivo recopilar la mayor cantidad de información relevante sobre el comportamiento modal del edificio. En la Figura 3 y Figura 4 se muestra una de las alternativas de instrumentación, basada en acelerómetros triaxiales distribuidos en seis plantas del edificio. Las señales de eventos registrados por los sensores constituyen el insumo para la aplicación de la metodología del análisis modal operacional OMA mediante el uso de los métodos FDD y el EFDD para obtener los parámetros modales $\mathrm{y}$, a partir de su análisis, realizar la monitorización de salud estructural del edificio. En el caso de la terraza se propone la ubicación de dos sensores, en la Figura 5 se muestra la ubicación de los sensores en una vista de elevación.

El uso de sensores triaxiales reduce significativamente el número de sensores a utilizarse en un plan de instrumentación, debido a que posee 3 grados de libertad para la medición. Esto deriva en una mayor recolección de datos con un menor número de sensores desplegados, y está directamente asociado a una reducción de presupuesto en costos de instalación y en disponibilidad de equipos. Se recomienda instrumentar con al menos un sensor 
los pisos ubicados en la base y en la parte más alta del edificio y, además, un piso medio ubicado entre estos dos (Celebi, 2000). Por estas razones y debido a la tipología poco convencional de la edificación, se implementa la propuesta que se detalla en la Tabla 1. De este modo, se propone el despliegue de un total de 18 sensores distribuidos en seis pisos del edificio, en concreto cuatro sensores para el subsuelo y la primera planta, mientras que, para los demás pisos superiores dos sensores por piso.

\section{Figura 3}

Localización de Sensores, de los Pisos: Tercero, Quinto, Séptimo, Octavo

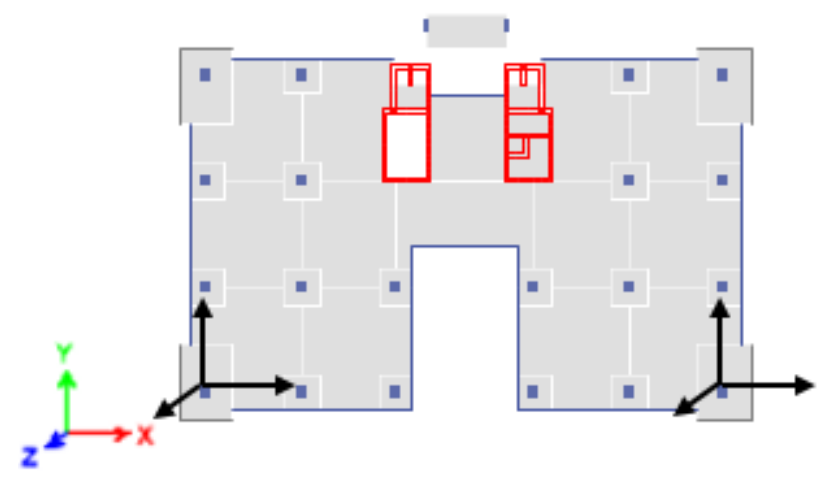

\section{Figura 4}

Localización de Sensores, de los Pisos: Subsuelo, Primero

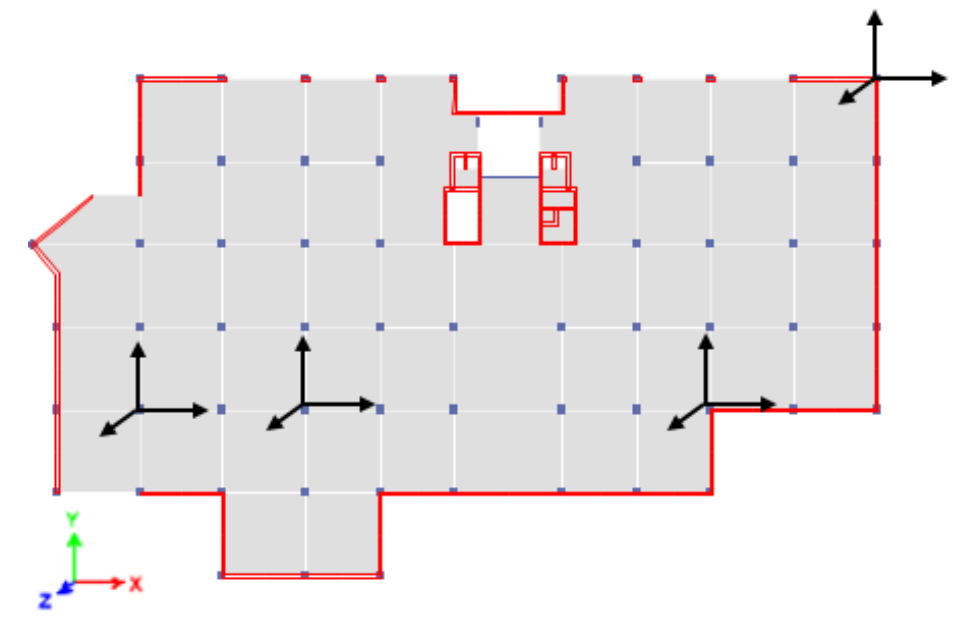

Tabla 1

Detalle de sensores instrumentados en la Edificación

\begin{tabular}{|c|c|c|c|c|}
\hline PLANTA & $\begin{array}{c}\text { SENSORES } \\
\text { COLUMNAS }\end{array}$ & $\begin{array}{c}\text { SENSORES } \\
\text { MUROS } \\
\text { ASCENSOR }\end{array}$ & $\begin{array}{c}\text { SENSORES } \\
\text { LOSA }\end{array}$ & $\begin{array}{c}\text { NÚMERO } \\
\text { TOTAL DE } \\
\text { SENSORES }\end{array}$ \\
\hline Subsuelo & 2 & 2 & 0 & 4 \\
\hline Primera & 2 & 0 & 2 & 4 \\
\hline Tercera & 2 & 0 & 0 & 2 \\
\hline Quinta & 2 & 0 & 0 & 2 \\
\hline Séptima & 2 & 0 & 0 & 2 \\
\hline Octava & 2 & 0 & 0 & 2 \\
\hline Terraza & 0 & 2 & 0 & 2 \\
\hline
\end{tabular}




\section{Arquitectura Tecnológica}

Respecto a la arquitectura tecnológica, con el objetivo de monitorizar los eventos sísmicos en el edificio objeto de estudio, se despliega una red de sensores inalámbricos (WSN) con las características de: escalabilidad, sencilla instalación en edificaciones existentes, detección automática de eventos sísmicos y envío de datos a la nube para el acceso remoto.

La arquitectura se basa en el modelo cliente-servidor, donde el servidor consiste en un nodo principal o gateway y los clientes corresponden a múltiples estaciones de acelerógrafos. Además, en el nodo gateway se implementa un sistema automático de detección de eventos sísmicos, el cual detecta en tiempo real un evento y solicita a las estaciones de acelerógrafos las muestras de dicho evento. Por último, estas estaciones suben un archivo con los datos del evento a la nube. Para la comunicación entre los nodos se despliega una red tipo ad hoc o no estructurada, donde cada dispositivo puede actuar ya sea como enrutador o cliente, lo que permite una mayor tolerancia frente a fallos de conectividad, en (Conti \& Giordano, 2014), se discuten la principales ventajas y desafíos para dicha tecnología. Además, se emplea el protocolo MQTT (Message Queuing Telemetry Transport), el cual es un mecanismo ampliamente empleado para la gestión de dispositivos IoT con alto nivel de seguridad y bajo requerimiento de ancho de banda (Yang et al., 2019).

En la Figura 5, se presenta la topología de la red, la ubicación aproximada de los sensores en la edificación objeto de estudio y los equipos implementados. Como se muestra en la figura, se desarrollan dos tipos de estaciones de acelerógrafos. La primera estación se basa en el trabajo presentado en (Muñoz et al., 2021), la misma que emplea un dispositivo SBC (Single Board Computer) y un microcontrolador que realiza la lectura de los datos del sensor acelerómetro, la sincronización de tiempo y el envío de datos al dispositivo SBC. El segundo tipo de estación, denominada estación nodo concentrador-sensores, corresponde a una variante de la primera estación. La cual dispone de un nodo concentrador con la SBC y múltiples nodos sensores que se comunican con el concentrador mediante una red cableada y el estándar RS485 .

\section{Figura 5}

Diagrama de la Arquitectura Tecnológica Implementada en el Edificio Objeto de Estudio

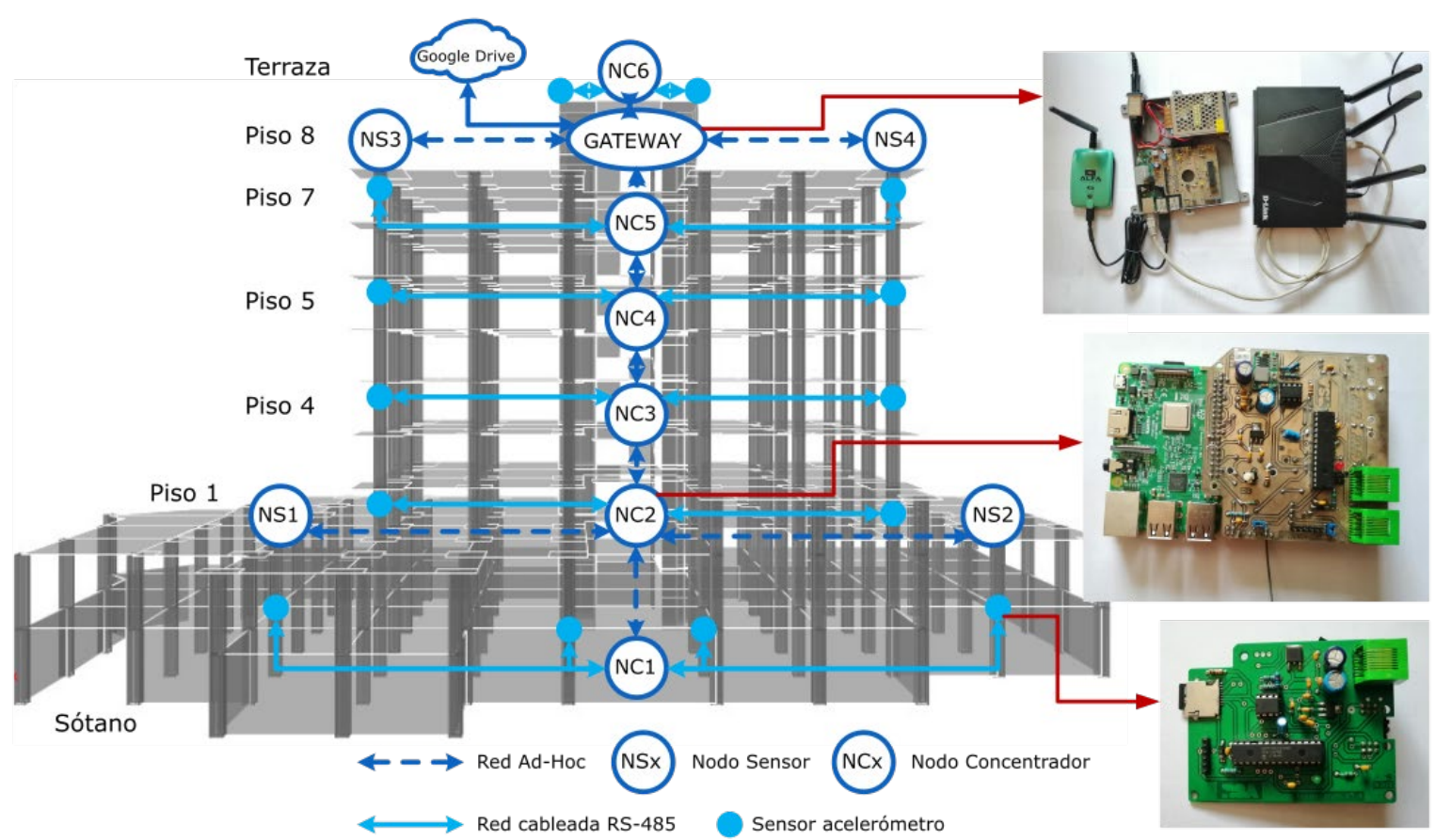


Por otro lado, el nodo gateway también se basa en el sistema de los nodos sensores. Sin embargo, con el objetivo de acceder remotamente a la información sísmica, en tiempo real y con un bajo consumo de ancho de banda, se instala un router 4G LTE para proporcionar acceso a Internet a la red de sensores y se implementó un sistema automático de detección de eventos sísmicos. Este sistema se basa en el algoritmo STA/LTA (Short Term Average to Long Term Average) recursivo (Azam Sya'bani et al., 2020), el cual tiene las ventajas de sencillez y eficiencia computacional.

Adicionalmente, para levantar la red de comunicación, se instala un módulo wifi externo en el dispositivo SBC de cada nodo. En particular, dado que no requiere una infraestructura prexistente, se despliega una red ad hoc multisalto con topología lineal y se emplea el protocolo MQTT para la comunicación entre las estaciones. Además, ya que los nodos se encuentran en posiciones fijas en el edificio, se definen direcciones IP estáticas y rutas estáticas para cada estación.

Finalmente, el diagrama funcional con la secuencia de la comunicación entre las estaciones se presenta en la Figura 6. Como se puede apreciar, en primera instancia, el dispositivo gateway lee los datos del sensor acelerómetro en tiempo real. Luego, el nodo principal aplica un filtro FIR (Finite Impulse Response) y calcula el valor de STA/LTA recursivo. A continuación, con el valor calculado de STA/LTA se analiza si corresponde a un evento sísmico. En caso afirmativo, el dispositivo gateway publica en un tópico MQTT la información del evento detectado y las estaciones de acelerógrafos suscritas a este tópico reciben dicha información. Luego, las estaciones solicitan a cada nodo sensor las muestras del evento detectado. Finalmente, cada estación crea un archivo con los datos de cada sensor y lo sube a la nube, específicamente a Google Drive, para el acceso remoto y post procesamiento de la información.

\section{Figura 6}

Diagrama Funcional de la Comunicación entre las Estaciones

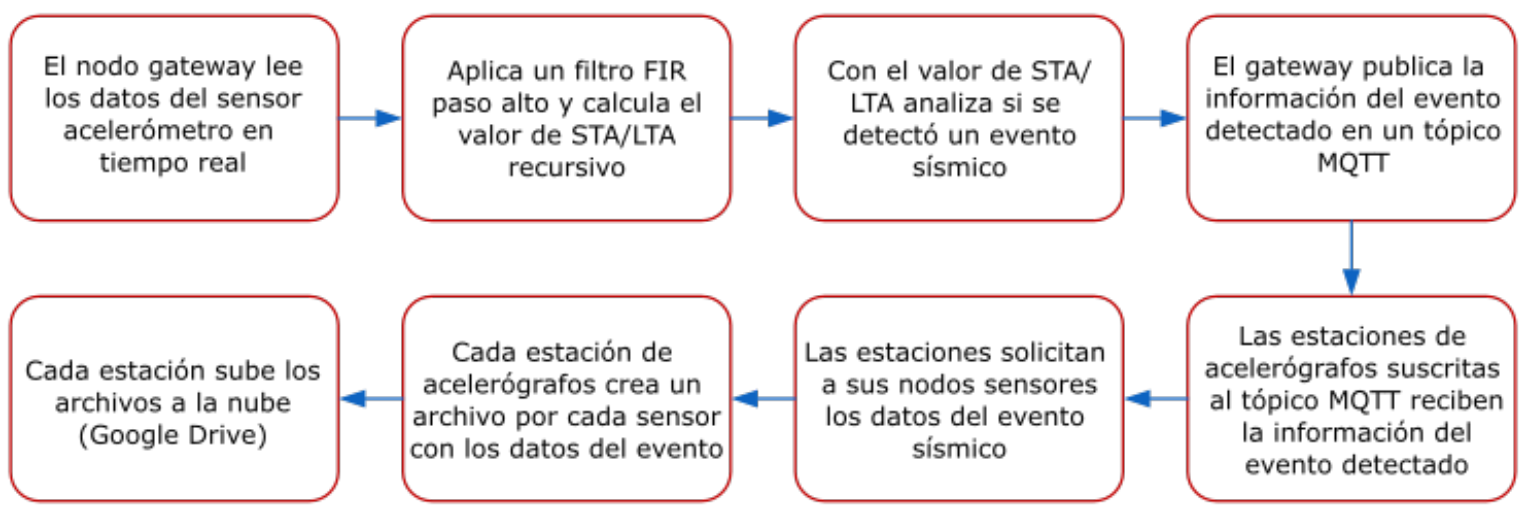

\section{Procesamiento de Datos}

El procesamiento de datos medidos por los sensores, consiste inicialmente en la transformación de valores binarios a valores de aceleración $\left(\mathrm{cm} / \mathrm{s}^{2}\right)$ para cada uno de los canales. Posteriormente, se separan estos datos de aceleración en sus 3 ejes. Finalmente, se realiza el proceso del cálculo de los parámetros modales de la estructura, mediante la aplicación de la metodología del OMA.

El Análisis Modal Operacional (OMA) se enfoca en la identificación de los parámetros modales de una edificación; este análisis deriva en un modelo modal en el que solo se usan respuestas medidas y sin conocer de manera explícita la excitación de entrada (Greiner, 2009). 
Los parámetros modales a identificar en la estructura son las frecuencias naturales de vibración y los modos de vibración. Para el análisis se emplean los métodos FDD y EFDD, el segundo es una versión mejorada del primero. Este enfoque estima los modos de vibración a partir de las densidades espectrales de potencia (PSD, Power Spectral Density) calculadas en la condición de una entrada de ruido blanco y una estructura ligeramente amortiguada.

Además, se emplea el criterio de aseguramiento modal MAC (Modal Assurance Criterion), el cual se define como un valor escalar constante que relaciona el grado de linealidad entre dos modos de referencia (Allemang, 2003). A continuación, se presentan los dos métodos analizados y los respectivos procedimientos matemáticos empleados en su aplicación:

\section{Descomposición en el Dominio de la Frecuencia (FDD)}

El método FDD se desarrolla en el dominio de la frecuencia y estima los modos utilizando una descomposición de valores singulares (SVD, Singular Value Decomposition) de cada una de las matrices PSD. Esta descomposición corresponde a una identificación de un sistema de un grado de libertad (SDOF) para cada valor singular.

A continuación, se detallan las relaciones matemáticas más importantes para comprender la técnica FDD y el algoritmo utilizado en el análisis. En la Figura 7, se presenta el procedimiento empleado para la descomposición en el dominio de la frecuencia; este método parte del modelo matemático que relaciona entradas desconocidas $(\mathrm{x}(\mathrm{t}))$ y respuestas medidas $(\mathrm{y}(\mathrm{t}))$, y se expresan mediante la ecuación planteada en (Brincker et al., 2000), la cual involucra variables como: la matriz de densidad espectral de entrada y salida, número de sensores y la matriz función de respuesta en frecuencia (RFR, Frecuency Response Function).

La matriz de densidad espectral PSD se genera aplicando la transformada de Fourier a los datos medidos por los sensores; con esto se indica la potencia de una señal distribuida en el espectro de las frecuencias, mediante la siguiente expresión:

$$
G_{y y}(j w)=\sum_{k=1}^{m} \frac{d_{k} \phi_{k} \phi_{k}^{T}}{j w-\lambda_{k}}+\frac{\bar{d}_{k} \bar{\phi}_{k} \bar{\phi}_{k}^{T}}{j w-\bar{\lambda}_{k}} \quad \text { Eq.1 }
$$

Donde, $G_{y y}(j w)$ es la matriz PSD de canales de respuesta medidos, $m$ indica solo los modos dominantes, $\phi_{k}$ denota el vector de forma modal, $\bar{\lambda}_{k}$ es un valor complejo que contiene frecuencias y amortiguamiento, " $T$ " indica la transpuesta del vector y el subíndice "-" es la conjugada compleja.

El diagrama funcional presentado en la Figura 7 esquematiza el algoritmo de los métodos FDD y EFDD (Farshchin, 2015). En la primera etapa, se realiza el preprocesamiento de los dados de aceleraciones del evento sísmico medido por los sensores. Luego, se desarrolla el cálculo de la matriz PSD a partir de la expresión matemática Gyy. El proceso continúa con la descomposición de la matriz PSD por medio de los valores singulares SVD. Una vez calculada la matriz, se genera el gráfico espectral del primer valor singular de la matriz PSD y las frecuencias, y posteriormente, se efectúa la selección manual de los picos de frecuencias para cada eje dependiendo del número de parámetros que se deseen obtener. Finalmente, se obtienen los parámetros modales correspondientes a frecuencias y modos de vibración en forma de tablas. 


\section{Figura 7}

Flujograma de Metodología Empleada en los Métodos FDD y EFDD

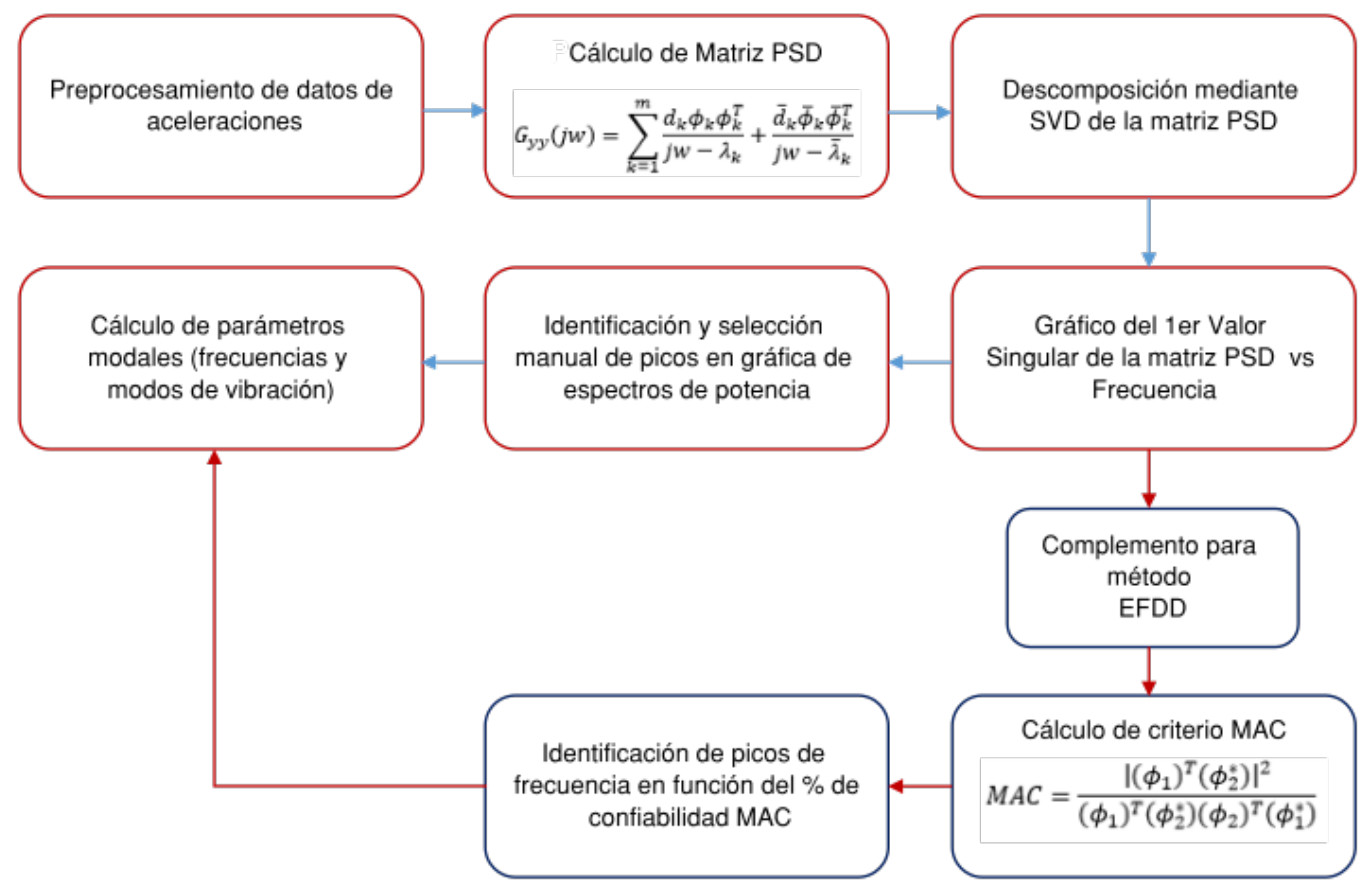

\section{Descomposición en el Dominio de la Frecuencia Mejorado (EFDD)}

El método EFDD corresponde a una mejora del método FDD (Jacobsen et al., 2007), en este se incluye un análisis de seguridad mediante la inclusión del criterio de aseguramiento modal MAC (Allemang, 2003). Este criterio relaciona dos modos a base de la siguiente expresión:

$$
M A C=\frac{\left|\left(\phi_{1}\right)^{T}\left(\phi_{2}^{*}\right)\right|^{2}}{\left(\phi_{1}\right)^{T}\left(\phi_{2}^{*}\right)\left(\phi_{2}\right)^{T}\left(\phi_{1}^{*}\right)} \quad \text { Eq.2 }
$$

Donde, $\phi_{1}$ corresponde al primer vector modal, $\phi_{2}$ al segundo vector modal compatible, el subíndice " $\mathrm{T}$ " denota la transpuesta del vector, y el signo "*”" corresponde a una matriz compleja conjugada.

Específicamente, el criterio MAC obtiene valores escalares entre 0 y 1 , presentados en forma de matriz. Valores de 1 o cercanos, denotan una buena configuración o una relación consistente entre estos, es decir son idénticos; mientras que valores de 0 o cercanos denotan una configuración deficiente o no consistente, son modos ortogonales. Es decir, se considera la ortogonalidad entre dos modos adyacentes. La variable $\phi$ denota los parámetros modales correspondientes a los vectores de formas modales.

El procedimiento a seguir es el mismo que en el método FDD, con la variación en el proceso luego de generar el gráfico del primer valor singular, como se indica en la Figura 7. A partir de aquí, el análisis continúa con el cálculo del criterio MAC entre los picos de resonancia y sus líneas de frecuencia vecinas, de esta manera se obtiene una auto correlación de las funciones para cada modo del sistema. Posteriormente, a base del valor de seguridad de MAC ( 0.8 como valor mínimo, 0,9 valor recomendado) definido por el usuario se identifican los picos en la gráfica y finalmente, se calculan los parámetros modales de la estructura. En la siguiente sección se presentan los resultados de la investigación realizada. 


\section{Resultados}

En esta sección se muestra la evaluación de las propiedades mecánicas del hormigón, datos de los acelerogramas medidos y procesados en los tres ejes, así como los parámetros modales obtenidos a partir del análisis modal operacional (OMA) utilizando los métodos de análisis FDD y EFDD.

En primer lugar, se realiza la evaluación de las propiedades mecánicas del hormigón mediante los ensayos planteados. Como se puede observar en la Tabla 2, el factor de corrección para las resistencias medidas entre esclerometría y rotura de núcleos de hormigón es de 1.02, debido a la correcta elección de la curva de correlación en el equipo Silver Schmidt $(10 \%$ percentil) el error obtenido fue únicamente del $2 \%$ el cual se corrige en los valores de resistencia obtenidas en el ensayo de esclerometría.

En la Tabla 3, se presentan los resultados de la resistencia a la compresión del hormigón de los elementos del edificio. Como se puede observar, las columnas del subsuelo y planta baja presentan un valor de resistencia mayor que en los demás pisos $\left(354 \mathrm{~kg} / \mathrm{cm}^{2}\right.$ en el sótano y 218 $\mathrm{Kg} / \mathrm{cm}^{2}$ en la cuarta planta). Además, se indican los valores obtenidos para los muros de hormigón y el macizado de las losas en los pisos ensayados. Cabe mencionar que el hormigón de los elementos del edificio no se encontraba distribuido de manera uniforme en el elemento, motivo de las diferencias marcadas entre resistencias.

\section{Tabla 2}

Factor de Corrección de Curva de Correlación Esclerométrica (Samaniego, 2020)

\begin{tabular}{|c|c|c|c|c|c|}
\hline & \multicolumn{2}{|c|}{ ESCLERÓMETRO } & NÚCLEOS & & \\
\hline $\begin{array}{c}\text { COLUMNA } \\
\#\end{array}$ & $\begin{array}{c}\text { REBOTE } \\
\text { PROMEDIO }\end{array}$ & $\begin{array}{c}\text { RESISTENCIA } \\
\left(\mathrm{Kg} / \mathrm{cm}^{2}\right)\end{array}$ & $\begin{array}{c}\text { RESISTENCIA } \\
\left(\mathrm{Kg} / \mathrm{cm}^{2}\right)\end{array}$ & $\begin{array}{l}\text { FACTOR DE } \\
\text { CORRECCIÓN }\end{array}$ & \\
\hline 8 & 51.3 & 330 & 171.61 & 0.520 & \\
\hline 52 & 50.3 & 315 & 324.26 & 1.029 & 102 \\
\hline 53 & 50.3 & 315 & 318.50 & 1.011 & \\
\hline 53.1 & 50.3 & 315 & 292.65 & 0.929 & \\
\hline
\end{tabular}

Tabla 3

Resistencia a la Compresión Promedio de Elementos de Hormigón (Samaniego, 2020)

\begin{tabular}{|c|c|c|c|}
\hline \multicolumn{4}{|c|}{ RESISTENCIA A COMPRESION $\left(\mathbf{K g} / \mathbf{c m}^{2}\right.$ ) } \\
\hline PISO & COLUMNAS & $\begin{array}{c}\text { MUROS } \\
\text { (ASCENSOR) }\end{array}$ & $\begin{array}{c}\text { LOSAS } \\
\text { (MACIZADO) }\end{array}$ \\
\hline Sótano & 354 & & \\
\hline Planta Baja & 340 & 332 & 495 \\
\hline Primera Planta & 261 & 408 & 464 \\
\hline Segunda Planta & 241 & 383 & 556 \\
\hline Tercera Planta & 219 & 372 & 541 \\
\hline Cuarta Planta & 218 & 408 & 372 \\
\hline Quinta Planta & 226 & 413 & 571 \\
\hline Sexta Planta & 224 & 408 & 531 \\
\hline Séptima Planta & 228 & 490 & \\
\hline Octava Planta & & & \\
\hline
\end{tabular}

Luego, se elige el sismo más relevante registrado por las estaciones instaladas en el edificio de estudio. El 26 de abril de 2021, se registra un evento sísmico de magnitud $3.9 \mathrm{Md}$ (magnitud de duración), el mismo se utiliza para el análisis gráfico y analítico. En particular, en la Figura 8, se presentan las gráficas de aceleración de los 3 ejes (longitudinal, transversal, vertical) de los sensores para 5 pisos del edificio. Como se puede observar, la amplitud de las 
aceleraciones varía en función del piso y es directamente proporcional a la altura del edificio. En consecuencia, a mayor altura se tiene mayor amplitud. Específicamente, para el eje longitudinal del 1er piso se tiene una magnitud RMS (Root Medium Square) de $0.203 \mathrm{~cm} / \mathrm{s}^{2}$, mientras que para el piso 7 la magnitud es de $0.223 \mathrm{~cm} / \mathrm{s}^{2}$.

\section{Figura 8}

Graficas de Aceleración para los ejes Longitudinal, Transversal y Vertical de 5 Pisos del Edificio

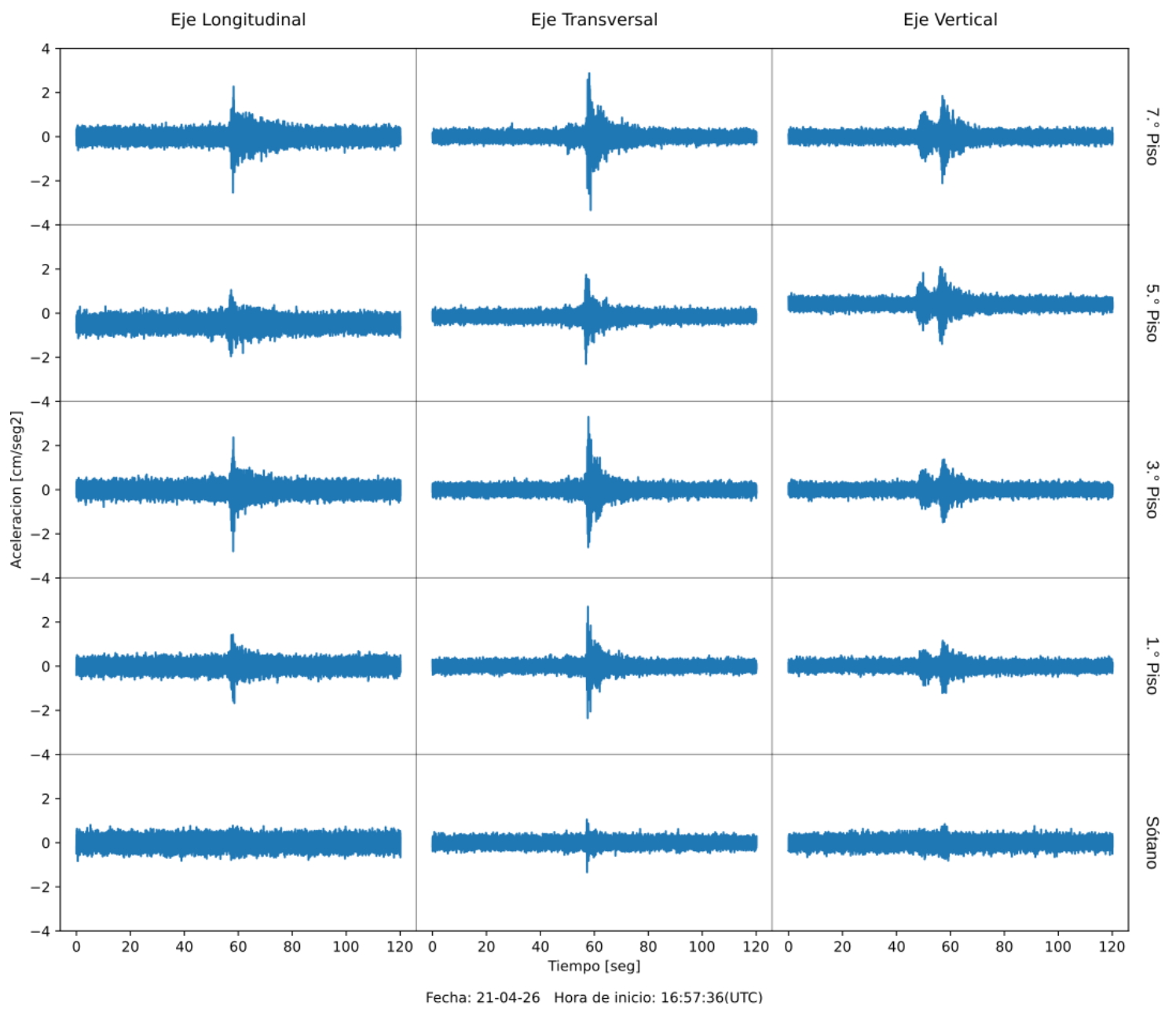

Por otro lado, se efectúa el análisis modal operacional mencionado en el apartado de metodología. Para tal objetivo se implementan los algoritmos de los métodos FDD y EFDD en Matlab. El análisis se realiza para los 3 ejes (longitudinal, transversal, vertical) y se grafican los datos de la matriz PSD como se muestra en la Figura 9. Por último, se seleccionan los picos en cada gráfica PSD para determinar los modos de vibración asociados a las frecuencias.

Continuando con el análisis OMA, como se indica en la figura mencionada, se seleccionan 10 picos para cada eje, debido a que se busca tener una muestra representativa de frecuencias a través de los modos fundamentales y modos altos de la estructura. Como se observa en la Figura 9, se determina una configuración espectral con un intervalo de $20 \mathrm{~Hz}$, para identificar de mejor manera los picos, con una ligera presencia de ruido. Con los picos de frecuencia claramente definidos e identificados se puede observar una configuración espectral con la presencia de ruido, lo cual dificulta la selección de picos, ya que se pueden cometer errores de criterio en este proceso. Cabe indicar que la selección de los picos de frecuencia se efectúa de forma manual en el método FDD. Para esto se realiza una inspección visual de cada 
gráfica de densidad espectral de potencia PSD, en consecuencia, esta selección está sujeta a criterios del investigador. Posteriormente, se aplica la metodología de la versión mejorada EFDD y se determina si dicha selección se emplea de manera correcta por medio del criterio MAC.

\section{Figura 9}

Gráficas de Densidad Espectral de Potencia y Picos Seleccionados para los Ejes Longitudinal, Transversal y Vertical
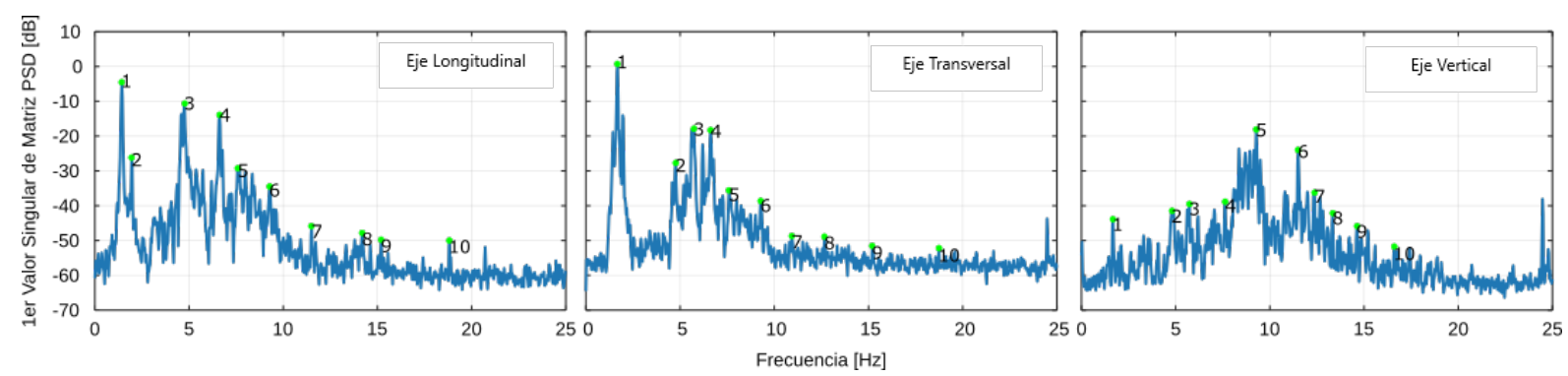

En cuanto a los resultados de este análisis, en la Tabla 4, se presentan los valores de frecuencia obtenidos para cada modo de vibración y su comparación con los valores numéricos obtenidos en el paquete computacional (ETABS). Mientras que, en la Tabla 5 se muestran los valores de MAC, se indica que de 10 valores de frecuencias analizados 7 tienen valores MAC aceptables (mayor a 0.80) para el eje longitudinal, mientras que 3 corresponden a una mala configuración en la selección de picos, siendo estos valores de frecuencia de $3.47 \mathrm{~Hz}, 7.59 \mathrm{~Hz}$ y $9.27 \mathrm{~Hz}$, teniendo además correspondencia entre otros modos de acuerdo a la matriz MAC.

Además, los resultados indican frecuencias compartidas en más de un eje (eje longitudinal y eje transversal). Esto significa la participación de modos de vibración en 2 ejes e implica un comportamiento no adecuado de la edificación. Finalmente, se observa que la frecuencia fundamental de la estructura medida con la instrumentación es de $1.43 \mathrm{~Hz}$ en el eje longitudinal, mientras que para el eje transversal es de $1.67 \mathrm{~Hz}$. En comparación con los resultados del modelo numérico la frecuencia fundamental en el eje longitudinal es de 1.367 $\mathrm{Hz}$ con una diferencia de $0.07 \mathrm{~Hz}(4.9 \%)$ respecto a frecuencia de las mediciones $(1.43 \mathrm{~Hz})$; de igual manera, la frecuencia fundamental para el eje transversal es de $1.65 \mathrm{~Hz}$ con una diferencia de $0.02 \mathrm{~Hz}(1.2 \%)$ respecto a las mediciones $(1.67 \mathrm{~Hz})$.

Tabla 4

Frecuencias Medidas con Sensores y Modelo Computacional

\begin{tabular}{|c|c|c|c|}
\hline \multirow{2}{*}{ MODO } & \multicolumn{2}{|c|}{ ANÁLISIS OMA } & \multirow{2}{*}{ MODELO } \\
\cline { 2 - 3 } & LONGITUDINAL & TRANSVERSAL & NUEÉRO \\
\cline { 2 - 3 } & $\begin{array}{c}\text { FRECUENCIA } \\
(\mathbf{H z})\end{array}$ & $\begin{array}{c}\text { FRECUENCIA } \\
(\mathbf{H z})\end{array}$ & $\begin{array}{c}\text { FRECUENCIA } \\
(\mathbf{H z})\end{array}$ \\
\hline 1 & 1.43 & 1.67 & 1.36 \\
\hline 2 & 1.95 & 4.76 & 1.65 \\
\hline 3 & 3.47 & 5.73 & 1.80 \\
\hline 4 & 4.76 & 6.19 & 4.95 \\
\hline 5 & 6.62 & 6.62 & 6.29 \\
\hline 6 & 7.59 & 7.59 & 7.35 \\
\hline 7 & 7.99 & 9.27 & 8.07 \\
\hline 8 & 8.36 & 10.92 & 8.62 \\
\hline 9 & 9.27 & 12.66 & 10.00 \\
\hline 10 & 11.5 & 15.19 & 10.20 \\
\hline
\end{tabular}


Tabla 5

Matriz de Valores MAC, Eje Longitudinal

\begin{tabular}{|c|c|c|c|c|c|c|c|c|c|c|}
\hline \multicolumn{10}{|c|}{ MAC } \\
\hline MODO & $\mathbf{1}$ & $\mathbf{2}$ & $\mathbf{3}$ & $\mathbf{4}$ & $\mathbf{5}$ & $\mathbf{6}$ & $\mathbf{7}$ & $\mathbf{8}$ & $\mathbf{9}$ & $\mathbf{1 0}$ \\
\hline $\mathbf{1}$ & 1.000 & 0.832 & 0.012 & 0.017 & 0.020 & 0.008 & 0.000 & 0.008 & 0.002 & 0.014 \\
\hline $\mathbf{2}$ & 0.832 & 1.000 & 0.852 & 0.551 & 0.516 & 0.008 & 0.138 & 0.034 & 0.044 & 0.050 \\
\hline $\mathbf{3}$ & 0.012 & 0.852 & 1.000 & 0.554 & 0.058 & 0.032 & 0.015 & 0.009 & 0.012 & 0.030 \\
\hline $\mathbf{4}$ & 0.017 & 0.551 & 0.554 & 1.000 & 0.824 & 0.051 & 0.015 & 0.020 & 0.004 & 0.003 \\
\hline $\mathbf{5}$ & 0.020 & 0.516 & 0.058 & 0.824 & 1.000 & 0.922 & 0.028 & 0.028 & 0.006 & 0.004 \\
\hline $\mathbf{6}$ & 0.008 & 0.008 & 0.032 & 0.051 & 0.922 & 1.000 & 0.754 & 0.423 & 0.802 & 0.764 \\
\hline $\mathbf{7}$ & 0.000 & 0.138 & 0.015 & 0.015 & 0.028 & 0.754 & 1.000 & 0.824 & 0.888 & 0.878 \\
\hline $\mathbf{8}$ & 0.008 & 0.034 & 0.009 & 0.020 & 0.028 & 0.780 & 0.824 & 1.000 & 0.851 & 0.823 \\
\hline $\mathbf{9}$ & 0.002 & 0.044 & 0.012 & 0.004 & 0.006 & 0.802 & 0.888 & 0.851 & 1.000 & 0.761 \\
\hline $\mathbf{1 0}$ & 0.014 & 0.050 & 0.030 & 0.003 & 0.004 & 0.764 & 0.878 & 0.823 & 0.761 & 1.000 \\
\hline
\end{tabular}

Cabe destacar que estos resultados corresponden a una evaluación inicial del comportamiento del edificio estudiado frente a un evento sísmico. En la Figura 10, se resaltan los primeros cuatro modos de vibración correspondientes al eje longitudinal, los cuales al ser modos compuestos (participación en más de un eje) en algunos casos y mantener formas inestables en la estructura denotan una respuesta inadecuada de la misma. Esto se debe a la configuración tipológica de la edificación, al ser un edificio de losas planas sin vigas soportada por columnas. En particular, un edificio sujeto a cargas sísmicas debe caracterizarse por una respuesta que no incluya los modos compuestos, al menos en sus 3 primeras configuraciones modales y dependiendo de la participación total de masa en estos modos.

\section{Figura 10}

Primeros 4 Modos de Vibración del Eje Longitudinal de la Estructura

Modo 1

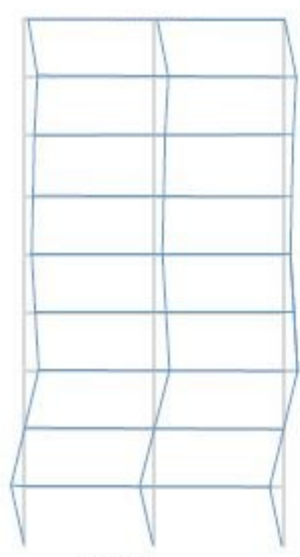

$1.43 \mathrm{~Hz}$
Modo 2

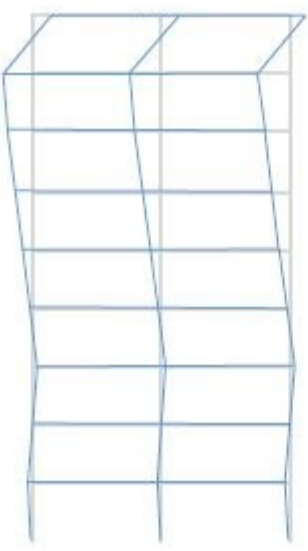

$1.95 \mathrm{~Hz}$
Modo 3

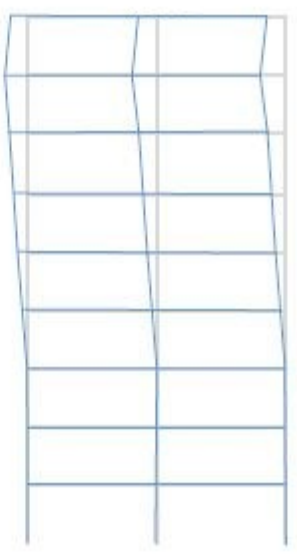

$3.47 \mathrm{~Hz}$
Modo 4

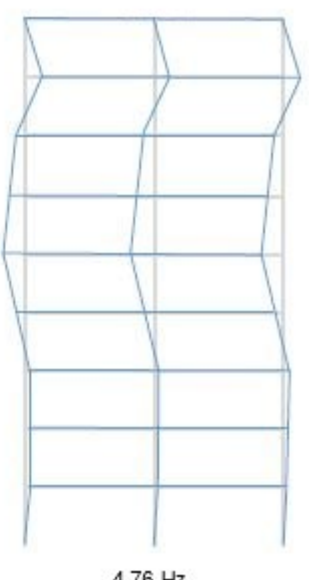

\section{Conclusiones}

En este artículo, se presenta una metodología que emplea mecanismos OMA, con el objetivo de obtener los parámetros modales de una edificación ante un evento que provoque daños (por ejemplo, un evento sísmico). Una ventaja de esta metodología es que se obtienen estos parámetros a base de datos de aceleraciones reales medidas in-situ en una estructura por 
medio de sensores y aplicando métodos de procesamiento de datos como el FDD y el EFDD. Se implementa un plan de instrumentación en una edificación existente de la ciudad de Cuenca (Ecuador) y se determinan valores de aceleración para el eje longitudinal con un RMS máximo de $0.223 \mathrm{~cm} / \mathrm{s}^{2}$ registrado en el piso 7 . Además, se ratifica que la amplificación de un sismo es de mayor magnitud en pisos superiores que en los pisos inferiores de una edificación.

De igual manera, la propuesta de instrumentación está sujeta a temas de logística en el edificio, por lo que dependiendo de los parámetros o tipos de modos de vibración que se busquen medir, se puede aplicar una propuesta que se adapte al caso necesario (por ejemplo, medir modos torsionales, medir modos en un determinado piso, entre otros).

La configuración modal calculada en la edificación evidencia un comportamiento no convencional de la estructura al encontrarse modos compuestos en la misma, debido a su configuración tipológica de losas planas. Además, se determinan los modos fundamentales que corresponden a frecuencias de $1.43 \mathrm{~Hz}$ y $1.67 \mathrm{~Hz}$ para los ejes longitudinal y transversal respectivamente. Un buen comportamiento de una estructura está definida por la configuración modal de los 3 primeros modos de vibración, los 2 primeros modos traslacionales con participación en un solo eje de movimiento, y el tercero a un modo de torsión.

Finalmente, con respecto a los métodos usados se evidencia que la versión mejorada EFDD reducen los errores al momento de la selección de los picos de frecuencia que el método FDD. Por lo tanto, mediante el método EFDD se estiman los parámetros modales con una precisión aceptable, ya que limitan los criterios del investigador al momento de la selección manual. De igual manera, se está trabajando en modelos de mayor alcance, como análisis modales analíticos no lineales y métodos automatizados de OMA, que servirán para optimizar las mediciones, reducir errores y complementar el estudio de los parámetros modales.

\section{Reconocimientos}

Este trabajo forma parte del proyecto de investigación "Tecnologías IoT y redes inalámbricas de sensores aplicados a la monitorización de salud estructural en edificios esenciales de la ciudad de Cuenca".

Se agradece al Departamento de RSA (Red Sísmica del Austro), a la Empresa Eléctrica Regional Centro Sur (EERCS) y a la Dirección de Investigación de la Universidad CuencaEcuador (DIUC), por el apoyo recibido y por los recursos facilitados durante el desarrollo de esta investigación.

\section{Referencias}

ACI 228.1R-03. (2003). In-Place Methods to Estimate Concrete Strength Reported. ACI Committee Reports, $228,1 R, 44$.

Allemang, R. J. (2003). The modal assurance criterion - Twenty years of use and abuse. Sound and Vibration, $37(8), 14-21$.

Arcentales, I. (2020). Evaluación De La Respuesta Dinámica Del Hospital Naval General Hosnag Sometido a Vibración Ambiental. Brain, 117(3), 607-636.

http://repositorio.espe.edu.ec/bitstream/21000/9434/1/T-ESPE-048572.pdf

ASTM C-805/C805M-18. (2018). Standard Test Method for Rebound Number of Hardened Concrete. ASTM International, 04.02, 1-4. http://www.astm.org/cgi-bin/resolver.cgi?C805C805M

ASTM C39 / C39M - 18. (2018). Standard Test Method for Compressive Strength of Cylindrical Concrete 
Specimens. ASTM International, 8. http://www.astm.org/cgi-bin/resolver.cgi?C39C39M

ASTM C42 / C42M - 18. (2018). Standard Test Method for Obtaining and Testing Drilled Cores and Sawed Beams of Concrete. ASTM International, 7. http://www.astm.org/cgi-bin/resolver.cgi?C42C42M

Azam Sya'bani, Y., Novianty, A., \& Luhur Prasasti, A. (2020). Implementation of Automatic First Arrival Picking On P-Wave Seismic Signal Using Logistic Regression Method. International Conference on Information and Comunication Technology (ICoICT), 8, 5-9.

Boscato, G., Dal Cin, A., Ientile, S., \& Russo, S. (2016). Optimized procedures and strategies for the dynamic monitoring of historical structures. Journal of Civil Structural Health Monitoring, 6(2), 265-289. https://doi.org/10.1007/s13349-016-0164-9

Brincker, R., Zhang, L., \& Andersen, P. (2000). Modal Identification from Ambient Responses using Frequency Domain Decomposition. MAC 18 : Proceedings of the International Modal Analysis Conference, 625630.

Cárdenas, E. M., \& Medina, L. U. (2021). Non-Parametric Operational Modal Analysis Methods in Frequency Domain: A Systematic Review. International Journal of Engineering and Technology Innovation, 11(1), 34-44. https://doi.org/10.46604/IJETI.2021.6126

Celebi, M. (2000). Seismic Instrumentation of Dams. USGS, 101(11), 1095-1104. https://doi.org/10.1061/ajgeb6.0000209

Celebi, M., Nishenko, S., Astill, C., \& Chung, R. M. (1998). Seismic Instrumentation of Federal Buildings a Proposal Document for Consideration By Federal Agencies.

Chopra, A. K. (2013). DYNAMICS OF STRUCTURES Theory and Applications to Earthquake Engineering. In PEARSON (Cuarta edi).

Conti, M., \& Giordano, S. (2014). Mobile ad hoc networking: Milestones, challenges, and new research directions. IEEE Communications Magazine, 52(1), 85-96. https://doi.org/10.1109/MCOM.2014.6710069

Farshchin, M. (2015). Frecuency Domain Decomposition (p. 1). https:/la.mathworks.com/matlabcentral/fileexchange/50988-frequency-domain-decomposition-fdd

Gkoktsi, K., \& Giaralis, A. (2019). A multi-sensor sub-Nyquist power spectrum blind sampling approach for low-power wireless sensors in operational modal analysis applications. Mechanical Systems and Signal Processing, 116, 879-899. https://doi.org/10.1016/j.ymssp.2018.06.049

Greiner, B. (2009). Operational Modal Analysis and its Application for SOFIA Telescope Assembly Vibration Measurements Operationelle. In Angewandte Chemie International Edition, 6(11), 951-952. Universidad de Stuttgart.

Hsiao, J. K. (2009). Computation of fundamental periods for moment frames using a hand-calculated approach. Electronic Journal of Structural Engineering, 9, 16-28.

Jacobsen, N. J., Andersen, P., \& Brincker, R. (2007). Using EFDD as a robust technique to deterministic excitation in operational modal analysis. Proceedings of the 2 nd International Operational Modal Analysis Conference, IOMAC 2007.

Kavitha, S., Joseph Daniel, R., \& Sumangala, K. (2016). Design and Analysis of MEMS Comb Drive Capacitive Accelerometer for SHM and Seismic Applications. Measurement: Journal of the International Measurement Confederation, 93, 327-339. https://doi.org/10.1016/j.measurement.2016.07.029

Lacanna, G., Ripepe, M., Marchetti, E., Coli, M., \& Garzonio, C. A. (2016). Dynamic response of the Baptistery of San Giovanni in Florence, Italy, based on ambient vibration test. Journal of Cultural Heritage, 20, 632-640. https://doi.org/10.1016/j.culher.2016.02.007 
Muñoz, M., Guevara, R., González, S., \& Jiménez, J. C. (2021). Reliable Data Acquisition System for a LowCost Accelerograph Applied to Structural Health Monitoring. Journal of Applied Science, Engineering, Technology, and Education, 3(2), 181-194. https://doi.org/10.35877/454ri.asci159

Muttillo, M., Battista, L. Di, De Rubeis, T., \& Nardi, I. (2019). Structural health continuous monitoring of buildings-A modal parameters identification system. 2019 4th International Conference on Smart and Sustainable Technologies, SpliTech 2019, 46-49. https://doi.org/10.23919/SpliTech.2019.8783051

NEC-SE-DS. (2015). Cargas Sísmicas Diseño Sismo Resistente. In Norma Ecuatoriana de la Construcción. http://www.indeci.gob.pe/proyecto58530/objetos/archivos/20110606102841.pdf\%0Ahttps:/www.habit atyvivienda.gob.ec/wp-content/uploads/downloads/2015/02/NEC-SE-DS-Peligro-Sísmico-parte-1.pdf (in spanish)

Neu, E., Janser, F., Khatibi, A. A., Braun, C., \& Orifici, A. C. (2016). Operational Modal Analysis of a wing excited by transonic flow. Aerospace Science and Technology, 49, 73-79. https://doi.org/10.1016/j.ast.2015.11.032

Ostachowicz, W., Soman, R., \& Malinowski, P. (2019). Optimization of sensor placement for structural health monitoring: a review. Structural Health Monitoring, 18(3), 963-988. https://doi.org/10.1177/1475921719825601

Pachón, P., Castro, R., García-Macías, E., Compan, V., \& Puertas, E. (2018). E. Torroja’s bridge: Tailored experimental setup for SHM of a historical bridge with a reduced number of sensors. Engineering Structures, 162(February), 11-21. https://doi.org/10.1016/j.engstruct.2018.02.035

Pachón, P., Infantes, M., Cámara, M., Compán, V., García-Macías, E., Friswell, M. I., \& Castro-Triguero, R. (2020). Evaluation of optimal sensor placement algorithms for the Structural Health Monitoring of architectural heritage. Application to the Monastery of San Jerónimo de Buenavista (Seville, Spain). Engineering Structures, 202(November 2019), 109843. https://doi.org/10.1016/j.engstruct.2019.109843

PROCEQ SA. (2016). Manual de operación SilverSchmidt y Hammerlink. 14.

PROCEQ SA. (2017). Manual de operación PROFOMETER.

Rojahn, C., Scawthorn, C., Anagnos, T., Wong, K., \& Poland, C. D. (2002). Rapid visual screening of buildings for potential seismic hazards, Handbook, FEMA 154. March, 1-140.

Samaniego, V. (2020). Análisis modal con orientación hacia monitorización de salud estructural. Caso edificio Empresa Eléctrica Regional Centrosur [Universidsad de Cuenca]. In Repositorio Institucional Universidad de Cuenca. http://dspace.ucuenca.edu.ec/handle/123456789/34869

Valenti, S., Conti, M., Pierleoni, P., Zappelli, L., Belli, A., Gara, F., Carbonari, S., \& Regni, M. (2018). A low cost wireless sensor node for building monitoring. EESMS 2018 - Environmental, Energy, and Structural Monitoring Systems, Proceedings, 1-6. https://doi.org/10.1109/EESMS.2018.8405827

Yang, J., Wu, P., Chen, Y., \& Wei, W. (2019). Development and Testing of the Earthquake Early Warning Information Push Platform Based on MQTT Protocol. Scientific Conference on Mechatronics Engineering and Computer Science (SCMC 2019), Scmc, 399-405. https://doi.org/10.25236/scmc.2019.082

Yun, D. Y., Kim, D., Kim, M., Bae, S. G., Choi, J. W., Shim, H. B., Hong, T., Lee, D. E., \& Park, H. S. (2021). Field measurements for identification of modal parameters for high-rise buildings under construction or in use. Automation in Construction, 121(October 2020), 103446. https://doi.org/10.1016/j.autcon.2020.103446

Zhou, Y., Zhou, Y., Yi, W., Chen, T., Tan, D., \& Mi, S. (2017). Operational Modal Analysis and Rational Finite-Element Model Selection for Ten High-Rise Buildings based on On-Site Ambient Vibration Measurements. Journal of Performance of Constructed Facilities, 31(5), 14. https://doi.org/10.1061/(asce)cf.1943-5509.0001019 